\title{
Do not count on me to imagine how I act: behavior contradicts questionnaire responses in the assessment of finger counting habits
}

\author{
Annalisa Lucidi • Catherine Thevenot
}

Published online: 11 February 2014

(C) Psychonomic Society, Inc. 2014

\begin{abstract}
The directionality of finger counting (i.e., from left to right or right to left) is supposed to compete with the orientation of the mental number line in determining number mental representations. Indeed, Western individuals who count on their fingers from right to left present a weaker SNARC effect than do individuals for whom the directionality of counting is the same as the mental number line. Observations of natural behavior should be the preferred methodology for classifying individuals according to their counting habits. Yet, to perform such classification, researchers usually rely on questionnaires or reports of imagined behaviors. However, we show in a series of three experiments that, on average, $26 \%$ of a sample of adults reported the opposite behavior from the one they actually implemented spontaneously when tested with an original ecological task. In a fourth experiment, this new task proved reliable, using a test-retest method. These results suggest that future studies about counting habits could benefit from the use of more ecological and functional tasks, rather than depending on noncontextualized questionnaires.
\end{abstract}

Keywords Syllable-counting task $\cdot$ Numerical cognition . Embodied cognition · Verbal reports · Verbal protocols

The "manumerical cognition" hypothesis states that the use of fingers in numerical activities during childhood shapes our comprehension of numbers (Fischer \& Brugger, 2011). This hypothesis is highly plausible, if we consider that using fingers to show numerosity universally precedes the use of more abstract codes, such as the Arabic or verbal ones (Butterworth, 1999). In fact, fingers are often seen as playing a functional

\footnotetext{
A. Lucidi $\cdot$ C. Thevenot $(\bowtie)$

FAPSE, University of Geneva, 40, bd du Pont D'Arve,

1205 Geneva, Switzerland

e-mail: catherine.thevenot@unige.ch
}

role in the development of numerical abilities (see Fayol \& Seron, 2005, for a review). This view stems from the observation that fingers constitute external aids to represent numbers, that they help in keeping track of number words in counting, and that they sustain the comprehension of the base-10 numerical system as well as the realization of basic arithmetic operations.

Moreover, according to Fischer (2008), the directionality of finger counting shapes the mental representation of numbers. More precisely, finger counting habits modulate spatial-numerical associations. Classically and in the Western culture, even for tasks in which magnitude is irrelevant (e.g., a parity judgment task), larger numbers are responded to faster with a right response key, whereas smaller numbers are responded to faster with a left one. The explanation given by Dehaene, Bossini, and Giraux (1993) is that the magnitude of a number is automatically activated and represented on a left-to-right-oriented mental number line. However, Fischer showed that the $66 \%$ of individuals who start counting from their left hand (i.e., leftstarters) present a stronger and more consistent "spatial-numerical association of response codes" (SNARC) effect than do the $34 \%$ of individuals who start counting on their right hand (i.e., right-starters). This result was independent of participants' handedness. The author concluded that counting habits compete with the left-to-right representation of numbers on the mental line and that the hand-digit mapping used in finger counting may also influence numerical processing.

In order to determine which hand is used first by individuals when they count on their fingers, Fischer (2008) initially used a paper-and-pencil questionnaire. A schematic drawing of two supine hands (thumb pointing outward) was presented to participants with the following instruction: "Imagine how you would count with your fingers from 1 to 10 ." Participants were then asked to write down the numbers next to the corresponding fingers of the two presented hands. In the same study, a few additional participants were asked to actually count on their 
fingers from 1 to 10 , and their responses were merged with those of the participants who filled out the questionnaire. In a subsequent study, Lindemann, Alipour, and Fischer (2011) used a computerized version of this task. Participants were first instructed to hold their empty hands in front of them in a supine position and to count aloud from 1 to 10 , using their fingers as they counted. Then, a schematic drawing of the two hands appeared on screen, together with ten input fields, one located next to each finger. The input field consisted of a drop-down menu with the numbers 1 to 10 . Participants had to remember how they counted with their fingers, and they were instructed to select the matching numbers in the corresponding input fields. The computerized task could be more appropriate than the written task because, as was noted by Fischer (2008), the written version could have induced a left-to-right bias due to reading and writing habits. However, Lindemann et al. showed that only five participants out of 52 differed in their responses on the different versions of the task (i.e., either in its paper-andpencil or computerized version). Therefore, a task wherein individuals are asked to imagine what they would do does not appear to be less reliable than a task wherein participants are asked to concretely count on their fingers.

Still, we think that the methodology used so far to study individuals' finger habits could be biased in several ways. First, it is well known in and outside the field of numerical cognition that reported behaviors do not always match actual ones (Barker, Fong, Grossman, Quin, \& Reid, 1994; Jensen, Potts, \& Jensen, 2005; Thevenot, Castel, Fanget, \& Fayol, 2010). Baumeister, Vohs, and Funder (2007) strongly encouraged researchers in psychology to base their conclusions on real behaviors rather than self-reports, hypothetical scenarios, or questionnaire ratings. The authors cleverly reminded us that "people have not always done what they say they have done, will not always do what they say they will do, and often do not even know the real causes of the things they do" (p. 397). Second, Fischer's tasks could be biased by the explicit request addressed to individuals. Indeed, Kirk and Ashcraft (2001) noted that instructions revealing the aim of an experiment might affect individuals' strategies. More precisely, behaviors that are implemented spontaneously for a specific purpose are likely to be different from the behaviors that are explicitly prompted by experimental instructions outside a natural context (Orne, 1962). Furthermore, as was noted by Fischer (2008) himself, the responses required from participants in his experimental design reflect memorized (or imagined) rather than actual counting. It is true that a potential caveat for Fischer's methodology is the fact that individuals have to remember what they did before reporting it. Of course, the necessary mental reconstruction of an action before report cannot be as trustworthy as direct observation of a natural behavior (Russo, Johnson, \& Stephens, 1989). Finally, the fact that within Fischer's methodology, the starting position for counting (i.e., supine positions of the hands) was always imposed on participants constitutes another argument against the ecological validity of the task. For all of these reasons, we thought that there was a need to design a more natural, ecological, contextualized, and functional implicit task in order to study finger counting habits.

The task that we developed is a counting task wherein participants have to determine the total number of syllables in a sentence directly after they have read it aloud. Because the phonological loop is blocked while reading the text (Baddeley, 1986), the best strategy to succeed is to keep track on fingers of the number of syllables already counted. In this task, spontaneous and natural behaviors are observed, because it is never mentioned to participants that they could use their fingers. Therefore, a great advantage of our task is that the attention of participants is never drawn to the object of the study. Moreover, the finger strategy is implemented spontaneously, without request from the experimenter, and finally, the fingers are used functionally for a justified purpose.

The present study, which consists of four experiments, aimed at testing the validity of the syllable-counting task. The goal of the first experiment was to compare the results obtained using Fischer's task with the results obtained using the syllablecounting task. As we already explained, we think that the syllable-counting task might be more appropriate for determining individuals' counting habits and, if we are right in assuming that the use of less ecological tasks is not optimum, a discrepancy in the results between the two methodologies might be revealed.

\section{Experiment 1}

\section{Method}

\section{Participants}

In order to homogenize our population, we selected 42 righthanded undergraduate students (11 males, 31 females; mean age $=20 ; 1$ years [i.e., 20 years, 1 month], $S D=2 ; 1$ ) from a sample of 47 students who were administered a handedness questionnaire adapted from Oldfield (1971). All of the students were French-speaking Swiss psychology students from the University of Geneva.

\section{Materials and procedure}

Participants were always presented with the syllable-counting task, followed by Fischer's questionnaire task. It was important to administer the syllable-counting task first because we did not want participants to guess its objective from the explicit questionnaire. Finally, before the end of the experiment, participants were presented with the handedness questionnaire. 
The syllable-counting task Participants were presented with four sentences (or expressions) that they had to read out loud. At the end of each sentence, they were asked to report how many syllables had been read. The sentences were presented in random order and were constituted of 5, 8, 13, or 16 syllables. The material was presented in French, as follows: Un lapin jaloux ("A jealous rabbit")-Les os du gros canard se cassent ("The bones of the big duck are breaking")-" Pas de printemps pour Marnie » est un excellent film ("Marnie is a great movie")-L'exposition au Grand Palais ne dure que jusqu'à fin Avril ("The exhibition at the Grand Palais lasts only until the end of April'). While participants were using their fingers during counting, the experimenter noted which hand was used first. Moreover, the precise order in which the fingers were used was also noted. Despite the fact that participants were not aware that the experimenter was observing their behaviors, no difficulty in coding arose, because the participants used clear, overt finger counting strategies. However, in order to maximize the reliability of the observations, the experimenter was seated next to the participant and could easily see his or her hands.

Fischer's questionnaire The exact same procedure as in Fischer (2008) was used here. As we already explained, a schematic drawing of two supine hands was printed and presented to participants. They were asked to imagine how they would count with their fingers from 1 to 10 and to write down the numbers next to the corresponding fingers of the two hands. A participant was considered as a left-starter when he or she wrote the numbers from 1 to 5 next to the left hand, and as a right-starter when he or she wrote the numbers 1 to 5 next to the right hand.

Handedness questionnaire Fourteen statements describing daily activities were presented to participants (e.g., "write" or "use a broom"), and they were asked to report which hand they would prefer in this activity (left, right, or both). A laterality index (LI) between -100 and +100 was calculated for each participant. Forty-three of the participants, who scored between 48 and 100, were classified as right-handed. Two of the participants, who scored between -100 and -28 , were classified as left-handed, and three participants who scored between -28 and 48 were considered ambidextrous. As was already stated above, only the data from the 42 right-handed participants were kept for the analyses.

Results

\section{Fischer's questionnaire}

Out of the 42 participants, 20 corresponded to left-starters (48\%) and 21 corresponded to right-starters (50\%). One participant could not be classified, because he did not perform the task correctly and was therefore not considered for the syllable-counting task.

\section{The syllable-counting task}

First of all, it is worth noting that, whatever the length of the sentences, all of our participants used their fingers to perform the task. The few participants who started the task without counting on their fingers quickly realized that it was not possible and promptly used them. The percentage of correct answers in the task was higher when five syllables were counted (100\%), rather than eight $(81 \%), 13(86 \%)$, or 16 $(73 \%), F(3,105)=4.61, p=.002 . \eta^{2}=.12$. However, no difference was apparent between those last three conditions, $F(2,70)=1.26, p=.29$.

Participants who started counting the syllables from their left hand for each of the four sentences were considered as left-starters. Conversely, participants who systematically started with their right hand were considered as rightstarters. Of our 41 participants, 16 corresponded to leftstarters $(39 \%)$ and 21 corresponded to right-starters $(51 \%)$. All participants started with their thumb. Four participants could not be classified as left- or right-starters, because they did not start with the same hand throughout the task. Percentages of correct responses in the counting task did not depend on the participant's finger counting habits $(79 \%$ and $81 \%$ for the left- and right-starters, respectively; $F<1$ ). The crucial results were that five of our left-starters reported starting with their right hand using Fischer's questionnaire, and eight of our right-starters reported starting with their left hand when presented with the questionnaire. Thus, 13 out of 37 participants clearly did not do what they thought they would do, which represents $35 \%$ of our sample (95\% confidence interval $=[.20, .51]$, which is significantly different from the 0 that would be expected if the two methodologies were perfectly consistent). We can therefore conclude that the proportion of participants who were not classified within the same category (i.e., left- or right-starter) using the two methodologies was significant at the $p<.05$ level. A power analysis conducted with the $G^{*}$ Power program (Erdfelder, Faul, \& Buchner, 1996) using classical criteria $(\alpha=.05$ and $\pi=.80$ ) showed that, for the test that we used, 25 participants would have been enough to obtain sensitive results. Indeed, the power analysis informed us that an effect size of .16 was required with our 42 participants, which is lower than the effect that we obtained.

\section{Discussion}

We showed here that 13 out of 37 participants reported the opposite behavior on Fischer's questionnaire from the one that they actually implemented spontaneously in our syllable- 
counting task. Interestingly, more participants reported starting with their left hand but actually used their right hand (i.e., eight participants) than the other way around (five participants). This might support Fischer's concern that his questionnaire could induce a left-to- right bias.

In addition to the 13 participants who showed incongruence across the tasks, four participants could not be classified as left- or right-starters in the syllable task because they did not start with the same hand throughout the experiment. This reveals another limitation of Fischer's questionnaire, which does not provide participants with the possibility to report the absence of preferential counting habits. On the contrary, the syllable-counting task identified $10 \%$ of participants with mixed behaviors, which constitutes a strong advantage over a mere binary classification (as either a left- or a right-starter). Considering participants as left- or right-starters according to Fischer's questionnaire, whereas they actually showed mixed behaviors, would lead to false inferences about their preferred directionality of counting.

Still, before strong conclusions can be put forward as to the validity of the syllable-counting task as a tool for the assessment of individuals' counting habits, several of its aspects have to be studied further. In this first experiment, participants had to count 5, 8, 13, or 16 syllables. Therefore, the number of syllables to be counted exceeded the range of finger counting on $50 \%$ of the trials. This departed from Fischer's questionnaire, wherein individuals were asked to report how they would count up to 10 on their fingers. This could explain the discrepancy in the results observed using his methodology and ours. In order to address this point, we carried out a second experiment and used new sentences of only five, eight, and ten syllables. Moreover, in this second experiment, we added up a debriefing phase wherein participants were asked whether or not they were aware of the object of the study. This was done to verify that our task does not draw the attention of participants on its real purpose. Indeed, as was already stated in the introduction, instructions revealing the aim of an experiment might affect individuals' strategies, and we expected our task to avoid this bias.

\section{Experiment 2}

Method

\section{Participants}

A group of 39 right-handed undergraduate students (three males, 36 females; mean age $=21 ; 3$ years, $S D=4 ; 11$ ) were selected from a sample of 47 students who were administered the same handedness questionnaire as in the previous experiment (Oldfield, 1971).

\section{Materials and procedure}

The tasks were administered in the same order as in the first experiment (i.e., syllable-counting task, handedness questionnaire, Fischer's questionnaire), and the debriefing phase was introduced at the end of the session.

The syllable-counting task Participants were presented with three sentences containing five, eight, or ten syllables, in a random order. As was already explained, and in contrast to the previous experiment, syllables could be counted without having to reuse one of the hands. In this version, the syllablecounting task was therefore closer to Fischer's questionnaire. The material was presented in French, as follows: La maison est bleue ("The house is blue")-Les carreaux du mur sont brisés ("The tiles on the wall are broken")-Un vélo en bon état se vend cher ("A bicycle in good condition can be expensive"). The experimenter noted which hand was used first, and the precise order in which fingers were used was also noted. The experimenter in this second experiment was not the same as the one in the first experiment.

Results

\section{Fischer's questionnaire}

Out of 39 participants, 16 corresponded to left-starters (41\%) and 23 corresponded to right-starters (59\%).

\section{The syllable-counting task}

One participant classified as a left-starter following Fischer's questionnaire did not use his fingers to perform the syllablecounting task and was excluded from the experimental sample. Interestingly, he was unable to perform the task and failed all trials, whatever the length of the sentences. Overall, the percentages of correct answers in the task did not depend on the number of syllables to be counted and corresponded to $87 \%$ for all three sentence lengths.

Participants who started counting the syllables from their left hand for each of the three sentences were considered leftstarters. Conversely, participants who systematically started with their right hand were considered right-starters. Out of our 38 participants, 11 corresponded to left-starters $(29 \%)$ and 23 corresponded to right-starters $(60 \%)$. All of them except one participant started with their thumb. The participant who did not behave like the others always started with the little finger. Four participants could not be classified as left- or rightstarters because they did not start with the same hand throughout the task. Moreover, three of our left-starters reported starting with their right hand using Fischer's questionnaire, and four of our right-starters reported starting with their left hand when presented with the questionnaire. Therefore, seven 
out of 34 participants showed behaviors opposite from the ones they reported, which represented $21 \%$ of our sample (95\% confidence interval of this proportion $=[.07, .34]$, which is different from 0 and includes the .15 effect size required, as calculated in a power analysis). As in the previous experiment, we can therefore conclude that the proportion of participants who are not classified within the same category using the two methodologies is significant at the $p<.05$ level. As we already mentioned, in addition to those seven participants, four others presented mixed behaviors in the syllablecounting task. Thus, 11 participants out of 38 would not have been classified correctly as left- or right-starters using Fischer's questionnaire, which represents $29 \%$ of the individuals tested for this experiment.

At the end of the session, participants were asked if they were aware of the purpose of the syllable-counting task. Out of the 47 participants tested, only three of them rightly spotted that we were studying finger counting strategies. The others mainly thought that the task was related to phonological awareness or to the simultaneous performance of two tasks.

\section{Discussion}

As in the previous experiment, we showed that a significant proportion of participants $(21 \%)$ presented opposite counting behaviors from the ones they reported using Fischer's questionnaire. In fact, almost $30 \%$ of the individuals who were asked to report their counting habits behaved differently when they spontaneously implemented finger counting procedures. These results were obtained despite the fact that the syllablecounting task remained in the finger counting range, and was therefore closer to Fischer's methodology. Moreover, our original results were replicated, even though the experimenter was not the same between Experiments 1 and 2. Therefore, systematic experimenter-related biases in the coding of the data or the inducement of specific behaviors cannot account for our results. Finally, we showed through a debriefing phase that, as we expected, the syllable-counting task did not draw the attention of participants to the purpose of the study, which could have biased the results (Kirk \& Ashcraft, 2001). Indeed, the vast majority of our participants thought that the object of the experiment was related to phonological awareness or the effects of concurrent tasks (i.e., counting and reading).

Thus, the syllable-counting task appears to be a good tool to assess individuals' counting habits and seems to be more appropriate than Fischer's questionnaire. Still, we have to test the possibility that our task was not more informative than directly asking participants to count on their fingers. Therefore, in the next experiment, we compared the results obtained with the syllable-counting task to the results collected in an enacted finger-counting situation.

We also investigated the possibility that participants' initial position has an influence on the choice of the starting hand.
Indeed, in Fischer's task, individuals have to imagine their hands in supine position. On the contrary, such an instruction could not be given in the syllable-counting task, wherein participants were not even asked to count on their fingers. Therefore, one potential explanation of the discrepancy in the results obtained using the two methodologies could be found in individuals' hand starting positions. In order to examine this possibility, participants in the following experiment were also presented with an enacted version of Fischer's questionnaire (adapted from Fabbri's, 2013, instruction: "Starting with your hands in a supine position, count on your fingers from 1 to 10 ").

\section{Experiment 3}

Method

\section{Participants}

A group of 33 right-handed undergraduate students (three males, 30 females; mean age $=21 ; 5$ years, $S D=8 ; 9$ ) were selected from a sample of 38 students who were administered the same handedness questionnaire as in the previous experiments (Oldfield, 1971).

\section{Materials and procedure}

Participants were presented with four different tasks. The syllable-counting task was always presented first and was systematically followed by Fischer's questionnaire in its classical version. Then, the order of the enacted version of the questionnaire and the enacted counting task was counterbalanced across participants. The handedness questionnaire was always administered between those last two tasks.

The syllable-counting task Participants were presented with four sentences containing five, seven, eight, or ten syllables in a random order. The numbers of syllables in the sentences were all in the range of finger counting, and the sentences were different from the ones used in the previous experiments. The material was presented in French, as follows: La chat est poilu ("The cat is hairy")-Le bébé dort dans son lit ("The baby is sleeping in his bed")-L'ordinateur est sous tension ("The computer is plugged in")-Un merveilleux cadeau est arrivé ("A fantastic present has been delivered"). The experimenter noted which hand was used first and the precise order in which fingers were used.

The enacted version of Fischer's questionnaire Participants were asked to count on their fingers from 1 to 10 , starting with their hands in a supine position. The experimenter 
concretely showed the participants how to position their hands before they started counting. The first hand used by participants to complete the task was noted.

The enacted counting task Participants were asked to count on their fingers from 1 to 10 , but there was no mention of a specific starting position. The experimenter noted which hand was used first by participants.

\section{Results}

\section{Fischer's questionnaire}

Out of 33 participants, 21 corresponded to left-starters (64\%) and 12 corresponded to right-starters (36\%).

\section{The syllable-counting task}

One participant classified as a left-starter following Fischer's questionnaire did not use his fingers to perform the syllablecounting task and was excluded from the experimental sample. Still, he managed to succeed at the task through mental counting, whatever the lengths of the sentences. Overall, the percentages of correct answers in the task did not depend on the number of syllables to be counted, $F<1$, and corresponded to $97 \%, 88 \%, 84 \%$, and $94 \%$ for five, seven, eight, and ten syllables, respectively.

Out of our 32 participants, 22 corresponded to left-starters $(69 \%)$ and 10 corresponded to right-starters (31\%). All of them started with their thumb. More importantly, four of our left-starters reported starting with their right hand using Fischer's questionnaire, and three of our right-starters reported starting with their left hand when presented with the questionnaire. Therefore, seven out of 32 participants showed behaviors opposite from the ones they reported, which represented $22 \%$ of our sample ( $95 \%$ confidence interval of this proportion $=[.08, .36]$, which is different from 0 and includes the .16 effect size required, as calculated in a power analysis). As in the previous experiments, we can conclude that the proportion of participants who were not classified within the same category using the two methodologies was significant at the $p<$ .05 level.

\section{Enacted finger-counting task}

Out of 32 participants, 16 participants (50\%) started with their left hand and 16 started with their right hand. However, nine of the participants who started with their right hand had actually used their left hand first in the syllable-counting task, and one participant who started with his right hand had been classified as a left-starter using the syllable-counting task. Therefore, ten participants out of 32 (95\% confidence interval of this proportion $=[.15, .47])$ did not show the same behavior when they were explicitly asked to count on their fingers and when they spontaneously implemented finger counting in an ecological situation.

\section{Enacted version of Fischer's questionnaire}

Out of 32 participants, 14 participants ( $44 \%$ ) started with their left-hand and 18 (66 \%) started with their right hand. Interestingly, only two participants who started with their right hand did not show the same preference in the enacted finger-counting task $(95 \%$ confidence interval of this proportion $=[-.02, .14]$, which did not differ from 0 ). Therefore, asking participants to start counting with their hands in a supine position or not does not make a significant difference in the way that they start counting

\section{Discussion}

The results of this experiment clearly show that explicitly asking individuals how to count or observing their natural behavior does not lead to the same conclusions about their left or right finger counting preference. Indeed, more than $30 \%$ of our participants showed opposite behaviors in an enacted finger-counting situation and in the syllable-counting task. This result strongly reinforces our conclusion that the syllable-counting task is a powerful tool to assess individuals' counting habits. It also provides further evidence for the fact that drawing the attention of participants to the object of the study can be quite detrimental for data collection (Kirk \& Ashcraft, 2001; Orne, 1962).

The second objective of the third experiment was to determine whether or not asking participants to start counting with their hands in a supine position could influence their behavior. This specific instruction was indeed given to participants in Fischer's task, but could not be stated within the syllable-counting task. However, our results clearly show that this does not affect individuals' responses. Indeed, asking or not asking our participants to start from a specific position did not significantly change the way they behaved.

Then, the results of the three experiments reported here have repeatedly shown that the syllable-counting task might be an interesting tool to determine individuals' counting habits. However, before affirming its validity, we need to provide evidence for its reliability. It is indeed important to ensure that our data can be replicated over time. Therefore, in the fourth experiment, the syllable-counting task was administered before and after a distracting task lasting $1 \mathrm{~h}$. We expected individuals' counting behaviors to be the same throughout the two testing sessions. 


\section{Experiment 4}

\section{Method}

\section{Participants}

Twenty-eight participants were tested for this experiment (four males, 24 females; mean age $=21 ; 2$ years, $S D=3 ; 7$ ). Because they were all right-handed, the complete sample was retained for the experiment.

\section{Materials and procedure}

The syllable-counting task was administered before and after a distracting task, which required participants to solve addition and comparison problems for $1 \mathrm{~h}$. The syllable-counting task was thus presented twice, and the same material and procedure were used as in Experiment 2.

\section{Results}

Two of the participants could not be retained in our experimental sample because one of them did not use his fingers in the syllable-counting task, and the other could remember the number of syllables in the sentences when retested after $1 \mathrm{~h}$. Out of the 26 remaining participants, following the first testing, six were classified as left-starters (23\%), 17 were classified as right-starters $(65 \%)$, and three participants showed mixed behaviors. Following the second testing, seven participants $(27 \%)$ were classified as left-starters and $19(73 \%)$ were classified as right-starters. In fact, only one participant who was classified as a left-starter during the first testing was classified as a right-starter following the second testing (95\% confidence interval of this proportion $=[-.03, .11]$, which did not differ from 0 ). Moreover, out of the three participants who showed mixed behaviors in the first session, one of them consistently used his right hand first in the second session, and two of them used their left hand first. If we consider that those three participants do not show the same behavior across sessions, the test-retest analysis barely failed to be reliable (95\% confidence interval of this proportion $=[.01, .29]$, which differed from 0 ). This will be discussed below.

\section{Discussion}

The results of this last experiment unambiguously provide evidence for the reliability of the syllable-counting task when individuals do not present mix behaviors. Indeed, only one participant who was tested twice using this task showed opposite behaviors over two sessions separated by $1 \mathrm{~h}$. Therefore, we can conclude that syllable counting constitutes a trustworthy tool for identifying left- or right-starters. However, three participants who showed mixed behaviors during the first session showed consistent behaviors when tested the second time. This suggests that individuals cannot be classified unequivocally as using indifferently their right or left hand on the basis of the syllable-counting task. Participants who present mixed behaviors should be retested to determine their prevailing preference. Another possibility would be to increase the number of sentences in the syllable task in order to infer the dominant behavior.

\section{General discussion}

In this study, we tested the validity of a new method conceived to assess individuals' counting habits. In the syllable-counting task, participants have to count the number of syllables in sentences while reading them. Because the phonological loop is blocked during the task, the best way to succeed is to keep track of the counted syllables on the fingers. We showed that this strategy was indeed adopted by almost all of our participants (i.e., 139 out of 142).

The great advantage of the syllable-counting task is that finger counting is implemented spontaneously by participants without an explicit request from the experimenter. Consequently, the attention of individuals is not drawn to the object of the study. In fact, our participants thought that the task was related to phonological awareness or to the effect of concurrent tasks rather than to finger counting.

Importantly, we showed repeatedly in three of our experiments that the natural behaviors that we observed using the syllable-counting task were not congruent with reported behaviors collected through Fischer's (2008) questionnaire, wherein participants have to imagine how they would count on their fingers from 1 to 10 and report the numbers on a schematic drawing. This was shown no matter how many syllables were to be counted (i.e., either within or above the range of finger counting). This was also true whatever the identity of the experimenter, which suggests that the coding of participants' behaviors was not ambiguous. However, to maximize the robustness of the syllable-counting task conclusions, further experiments could beneficiate from interrater coding, ideally using videotapes.

A more detailed look at our data reveals that $35 \%, 21 \%$, and $22 \%$ of the individuals in our samples indicated opposite behaviors using the syllable-counting task and Fischer's questionnaire in Experiments 1, 2, and 3. In fact, if we consider individuals who showed mixed behaviors in the syllablecounting task, only 76 participants out of 111 were consistently classified using the two methodologies. A correspondence analysis showed that this consistency between the two tests was weak $(K$ Cohen $=.36)$. Therefore, we showed repeatedly that individuals do not report what they concretely do when they spontaneously implement finger counting functionallyor in other words, for a justified purpose. Moreover, we showed in a fourth experiment that the syllable-counting task 
is a reliable method of investigation. Indeed, for participants who presented stable behaviors across conditions, similar results were obtained in two different sessions taking place before and after a 1-h distracting task. However, a limitation of the syllable-counting task was that the participants who showed mixed behaviors during the first testing showed systematic preferences during the second session.

We also showed in the third experiment that the results obtained with the syllable-counting task are not always consistent with the results obtained with a task wherein we simply asked participants to count on their fingers from 1 to 10 . As a matter of fact, $31 \%$ of individuals who started with one of their hands in this last task showed opposite behaviors in the syllable-counting task. This reveals that the advantage of the syllable-counting task cannot only be found in the fact that reports are not asked retrospectively. In that case, no discrepancy in the results would have been observed between the syllable-counting task and the task wherein participants are asked to count from 1 to 10 , which does not involve any retrospection. Therefore, merely drawing the attention of participants to the object of the study and making them "selfaware" of their behaviors changes the way that they behave, as compared to a situation that involves natural and spontaneous behavior. Another problem with directly asking participants to count on their fingers is that it elicits a behavior that is never produced in the real life. Indeed, finger counting is never implemented as a primary task, but always in order to achieve another goal, such as performing calculations or keeping track of counted concrete or abstract objects. This constitutes an additional strength of the syllable-counting task and further underlines its ecological dimension.

In the third experiment, we also showed that the discrepancy in the results between the syllable-counting task and the enacted counting task was observed whether or not participants were asked to start counting with their hands in a supine position. Therefore, the initial position does not seem to affect the way that individuals count on their fingers. This shows that the divergence in the results that we observed between Fisher's questionnaire and the syllable-counting task is not due to the different starting hand positions in those two tasks.

In conclusion, our results confirm that explicitly asking participants to count on their fingers or asking them to report their procedures does not provide a valid measure of their real behavior. Therefore, the present research puts in question the reasons behind the cross-cultural differences in counting habits that have been reported in the literature. As was mentioned in the introduction, between $66 \%$ and $68 \%$ of Scottish adults were classified as left-starters (Fischer, 2008; Lindermann et al., 2011). However, $69 \%$ of French adults (Sato \& Lalain, 2008) and $82 \%$ (Di Luca, Grana, Semenza, Seron, \& Pesenti, 2006) or $69 \%$ (Fabbri, 2013) of Italian adults were classified as rightstarters. Going even further than Previtali, Rinaldi, and Girelli (2011), who attributed those contradictory results to the use of different methods of investigation, we think that these contradictions could also be attributed to the fact that those methods are not always very ecological. Indeed, in all of those previous studies, participants were directly and explicitly requested to count on their fingers without concrete purpose, which, as is attested by our results, does not allow individuals to display their natural behavior.

Then, future studies concerning a possible influence of counting habits on the mental representation of numbers could benefit from the use of the syllable-counting task. For example, Fischer (2008) revealed a significant difference of the SNARC effect between left- and right-starters. More precisely, his results showed a typical and reliable SNARC effect for the left-starters, and no reliable SNARC effect for the rightstarters. In fact, Fischer's results reflected greater variability in spatial mapping in the group of right-starters than in the leftstarters. It might be possible to reduce this variability through a clearer classification of left- and right-starters using the syllable-counting task. It could therefore be envisioned that the SNARC effect would not only be absent, but actually reversed, in right-starters, which would strongly reinforce the view that finger habits can shape numerical mental organization and processing. Finally, a better classification of individuals as left- or right-starters could help in replicating Fischer's (2008) results. Indeed, despite a trend in the right direction, Tschentscher, Hauk, Fischer, and Pulvermüller (2012) failed to replicate the fact that right-starters do not show a SNARC effect. Moreover, whereas those authors nicely showed activation in the motor region contralateral to the starting hand when left-starters process numbers, they failed to demonstrate the same effects in right-starters. Even more disturbing is the fact that Fabbri (2013) found stronger SNARC effects in right-starters than in left-starters, which contradicted Fischer's results and line of reasoning. Again, a finer and more accurate identification of individuals' counting habits based on the syllable-counting task could allow researchers to obtain more coherent and reliable results. Nevertheless, further studies will be needed to put those assumptions to the test.

Author note We thank Michaela Chlostova for running the last three experiments reported in this article.

\section{References}

Baddeley, A. (1986). Working memory. Oxford, UK: Oxford University Press, Clarendon Press.

Barker, K., Fong, L., Grossman, S., Quin, C., \& Reid, R. (1994). Comparison of self-reported recycling attitudes and behaviors with actual behavior. Psychological Reports, 75, 571-577.

Baumeister, R. F., Vohs, K. D., \& Funder, D. C. (2007). Psychology as the science of self-reports and finger movement: What happened to actual behavior? Perspectives in Psychological Sciences, 2, 396403. 
Butterworth, B. (1999). The mathematical brain. London, UK: Macmillan.

Dehaene, S., Bossini, S., \& Giraux, P. (1993). The mental representation of parity and number magnitude. Journal of Experimental Psychology: General, 122, 371-396. doi:10.1037/0096-3445.122. 3.371

Di Luca, S., Grana, A., Semenza, C., Seron, X., \& Pesenti, M. (2006). Finger-digit compatibility in Arabic numeral processing. Quarterly Journal of Experimental Psychology, 5, 1648-1660.

Erdfelder, E., Faul, F., \& Buchner, A. (1996). GPOWER: A general power analysis program. Behavior Research Methods, Instruments, \& Computers, 28, 1-11. doi:10.3758/BF03203630

Fabbri, M. (2013). Finger counting habits and spatial-numerical association in horizontal and vertical orientations. Journal of Cognition and Culture, 13, 95-110.

Fayol, M., \& Seron, X. (2005). About numerical representations: Insight from neuropsychological, experimental, and developmental studies. In J. I. D. Campbell (Ed.), Handbook of mathematical cognition (pp. 3-22). New York, NY: Psychology Press.

Fischer, M. H. (2008). Finger counting habits modulate spatial-numerical associations. Cortex, 44, 386-392.

Fischer, M. H., \& Brugger, P. (2011). When digits help digits: Spatialnumerical associations point to finger counting as prime example of embodied cognition. Frontiers in Psychology, 2, 260.

Jensen, C., Potts, C., \& Jensen, C. (2005). Privacy practices of internet users: Self-report versus observed behaviors. International Journal of Human Computer Studies, 63, 203-227.

Kirk, E. P., \& Ashcraft, M. K. (2001). Telling stories: The perils and promise of using verbal reports to study math strategies. Journal of
Experimental Psychology: Learning, Memory, and Cognition, 27, $157-175$.

Lindemann, O., Alipour, A., \& Fischer, M. H. (2011). Finger counting habits in Middle Eastern and Western individuals: An online survey. Journal of Cross-Cultural Psychology, 42, 566-578. doi:10.1177/ 0022022111406254

Oldfield, R. C. (1971). The assessment and analysis of handedness: The Edinburgh inventory. Neuropsychologia, 9, 97-113. doi:10.1016/ 0028-3932(71)90067-4

Orne, M. T. (1962). On the social psychology of the psychological experiment: With particular reference to demand characteristics and their implications. American Psychologist, 17, 776-783.

Previtali, P., Rinaldi, L., \& Girelli, L. (2011). Nature or nurture in finger counting: A review on the determinants of the direction of numberfinger mapping. Frontiers in Psychology, 2, 363.

Russo, J. E., Johnson, E. J., \& Stephens, D. L. (1989). The validity of verbal protocols. Memory \& Cognition, 17, 759-769.

Sato, M., \& Lalain, M. (2008). On the relationship between handedness and hand-digit mapping in finger counting. Cortex, 44, 393-399.

Thevenot, C., Castel, C., Fanget, M., \& Fayol, M. (2010). Mental subtraction in high and lower-skilled arithmetic problem solvers: Verbal report vs. operand-recognition paradigms. Journal of Experimental Psychology: Learning, Memory, and Cognition, $36,1242-1255$.

Tschentscher, N., Hauk, O., Fischer, M. H., \& Pulvermüller, F. (2012). You can count of the motor cortex: Finger counting habits modulate motor cortex activation evoked by numbers. NeuroImage, 59, 3139 3148 . 